\title{
Computational modelling of wheat leaf growth and morphogenesis based on data from 3D LSM-images
}

\author{
U. Zubairova ${ }^{1 *}$, A. Doroshkov ${ }^{1,2}$ \\ ${ }^{1}$ Institute of Cytology and Genetics SB RAS, Novosibirsk, Russia \\ ${ }^{2}$ Novosibirsk State University, Novosibirsk, Russia \\ *e-mail: ulyanochka@bionet.nsc.ru
}

Key words: wheat leaf, epidermal patterning, computational modelling, confocal laser scanning microscopy, morphogenesis

Motivation and Aim: Leaf epidermis of a monocotyledonous plant is a widely used model system for studying morphogenesis. The epidermis of cereals (wheat, barley, rice) leaf is a complex tissue consisting of different cell types organizing in parallel cell rows. For such leaves, a unidirectional growth occurring for a long time enables to observe a series of successive morphogenetic stages at one snapshot. In this work, we propose the concept for using a growing wheat leaf to study dynamical changes in morphogenesis, including stress-induced changes. Linear leaf of a wheat, during its formation for a long time, maintains a phase of steady growth. Therefore, it is possible to observe a series of successive events of morphogenesis fixed in the cellular architecture of a mature leaf. Methods and Algorithms: High-resolution 3D LSM-images allow extracting quantitative characteristics describing the cellular structure of leaf epidermis. However, to obtain a large amount of statistical data methods of high throughput computer based image segmentation should be used. We developed a workflow for detection of structural properties of leaf epidermis from 3D images obtained from confocal laser scanning microscopy. The workflow includes the protocol of sample preparation, image processing ImageJ-plugin and data extraction algorithms. The data on the cellular architecture further will act as a basis for the elaboration and verification of spatial models accounting for structural features of leaves. For the leaf epidermis of cereals, a brickwork-like pattern combined with unidirectional growth allows to reduce the dimension and use a quasione-dimensional representation of the cellular ensemble in the model. This idea was realized in the model [1] growth of a linear leaf blade. The model allows for fitting of the visible cell length using the experimental cell length distribution along the longitudinal axis of a leaf epidermis.

Results: In this work, we assume a unidirectional growing cell ensemble starting from a meristem-like layer of generative cells and then generating parallel cell rows from every cell of the initial layer. We considered the growth zone of the leaf includes division and elongation zones; in addition, the division zone includes a zone of asymmetric divisions forming specialized cells (trichomes and stomata). The model was verified on qualitative and quantitative data on cold stress induced disturbances of morphogenesis in the epidermis of wheat leaf.

Acknowledgements: The work was supported by the project 17-44-543384 from Russian Foundation for Basic Research.

\section{References}

1. Zubairova U., Nikolaev S., Penenko A., Podkolodnyy N., Golushko S., Afonnikov D., Kolchanov N. (2016). Mechanical behavior of cells within a cell-based model of wheat leaf growth. Frontiers in plant science. 7:1878. 